\title{
Spectra and Photorelaxation of Hydroxyphenyl-Benzotriazole-Type UV Absorbers: From Monomers to Nanoparticles
}

\author{
Sergej Naumova, Bernd Herzog ${ }^{b}$ and Bernd Abela* \\ aLeibniz Institute of Surface Engineering (IOM), Permoserstr. 15, 04318 Leipzig, \\ Germany \\ bBASF Grenzach GmbH, Koechlinstr. 1, 79639 Grenzach-Wyhlen, Germany \\ * bernd.abel@iom-leipzig.de
}

To choose the reliable model for calculation of UV-Vis spectra of MBBT, the different levels of theory ware tested on the monomer molecule in dioxane (Fig. S1).

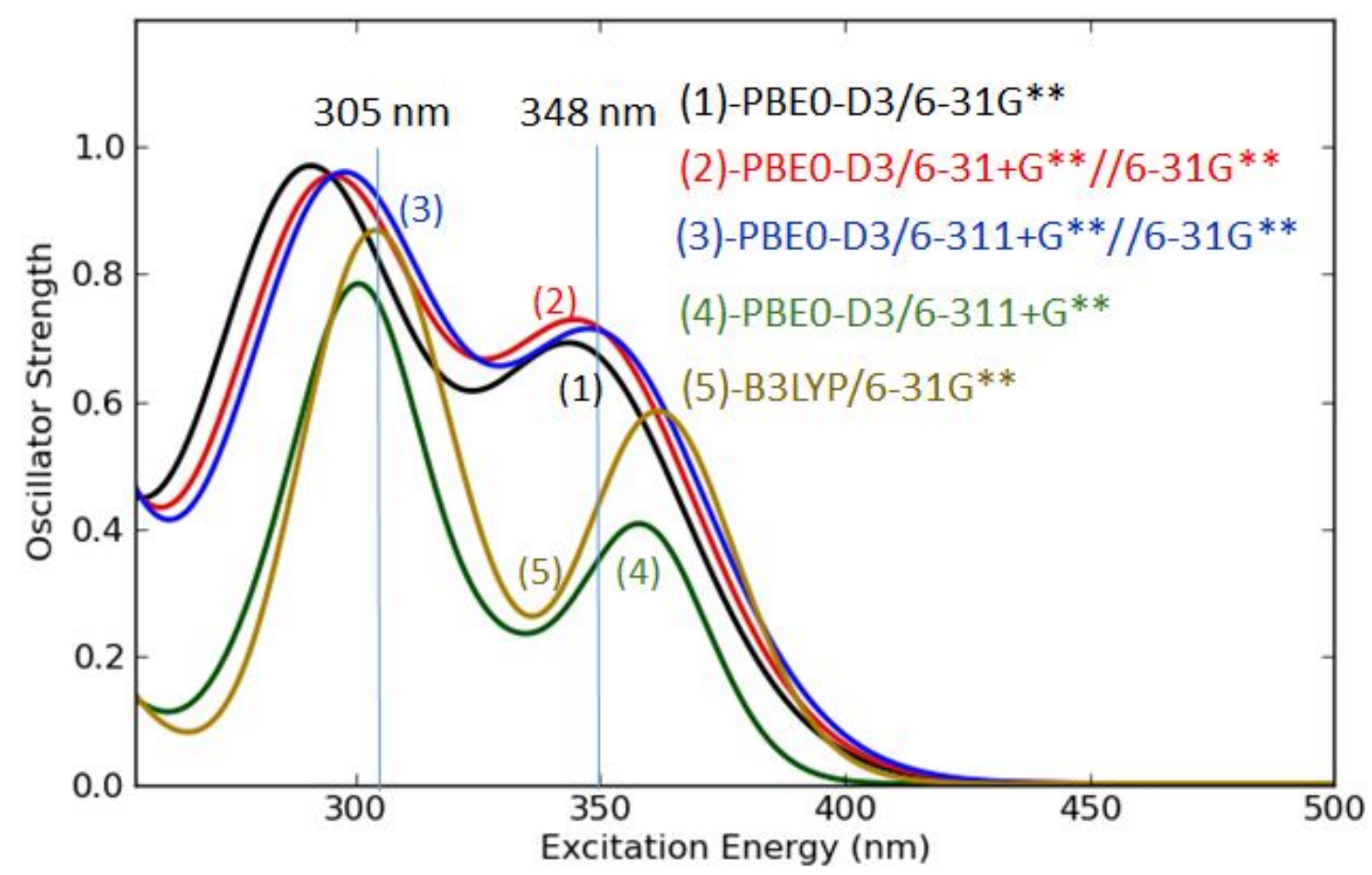

Figure S1. Comparison of UV-Vis of MBBT ground state molecules calculated in dioxane with TD DFT at different levels of theory. Here, $305 \mathrm{~nm}$ and $348 \mathrm{~nm}$ are experimental $\lambda_{\max }$ values in dioxane.

As can be seen, all computational models give reasonable agreement of the calculated UV/vis spectra in dioxane with experimental ones. The effect of a large basis set on the UV-Vis spectra at the structure optimised with middle level $6-31 \mathrm{G}^{* *}$ basis set (models 1,2 and 3 ) is rather small. The geometry optimisation with large $6-311+G^{* *}$ 
basis set (model 4) results in underestimation of low energy absorption band. However, the use of large basis set is very time consuming, especially by the study of the large systems, such as dimers. B3LYP functional with $6-31 \mathrm{G}^{* *}$ basis set (model 5 ) was tested for comparison. However, it underestimates the low energy absorption band too. As a consequence, the TD PBE0-D3/6-31G**/PBE level of theory was used throughout this study.
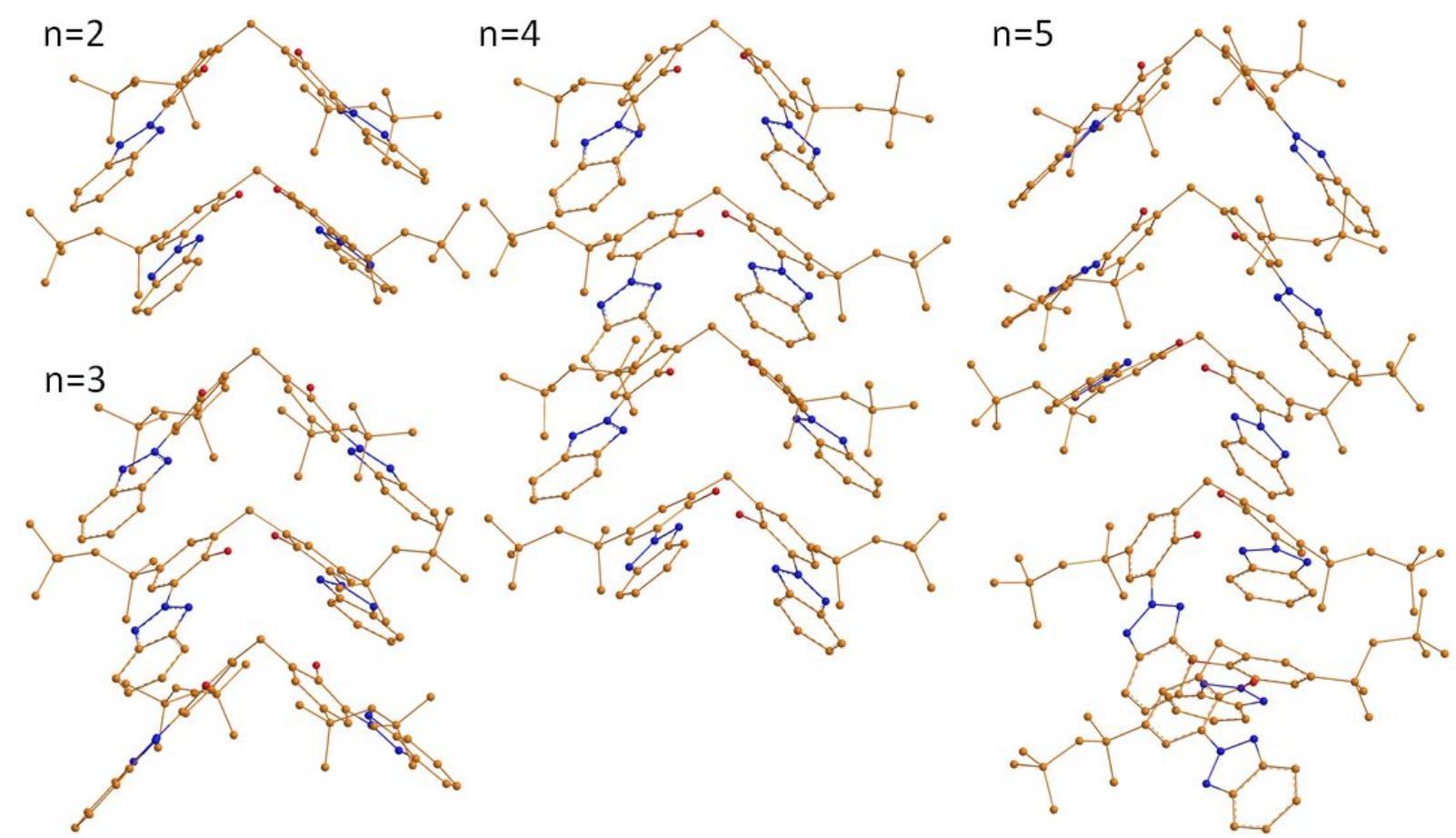

Figure S2. Possible structures optimised in water stable MBBT complexes with 2, 3, 4 and 5 units with mean stabilisation energy per one bond $\left(E_{s t}\right)$ about $100 \mathrm{~kJ} \mathrm{~mol}^{-1} . \mathrm{H}$ atoms are omitted. 


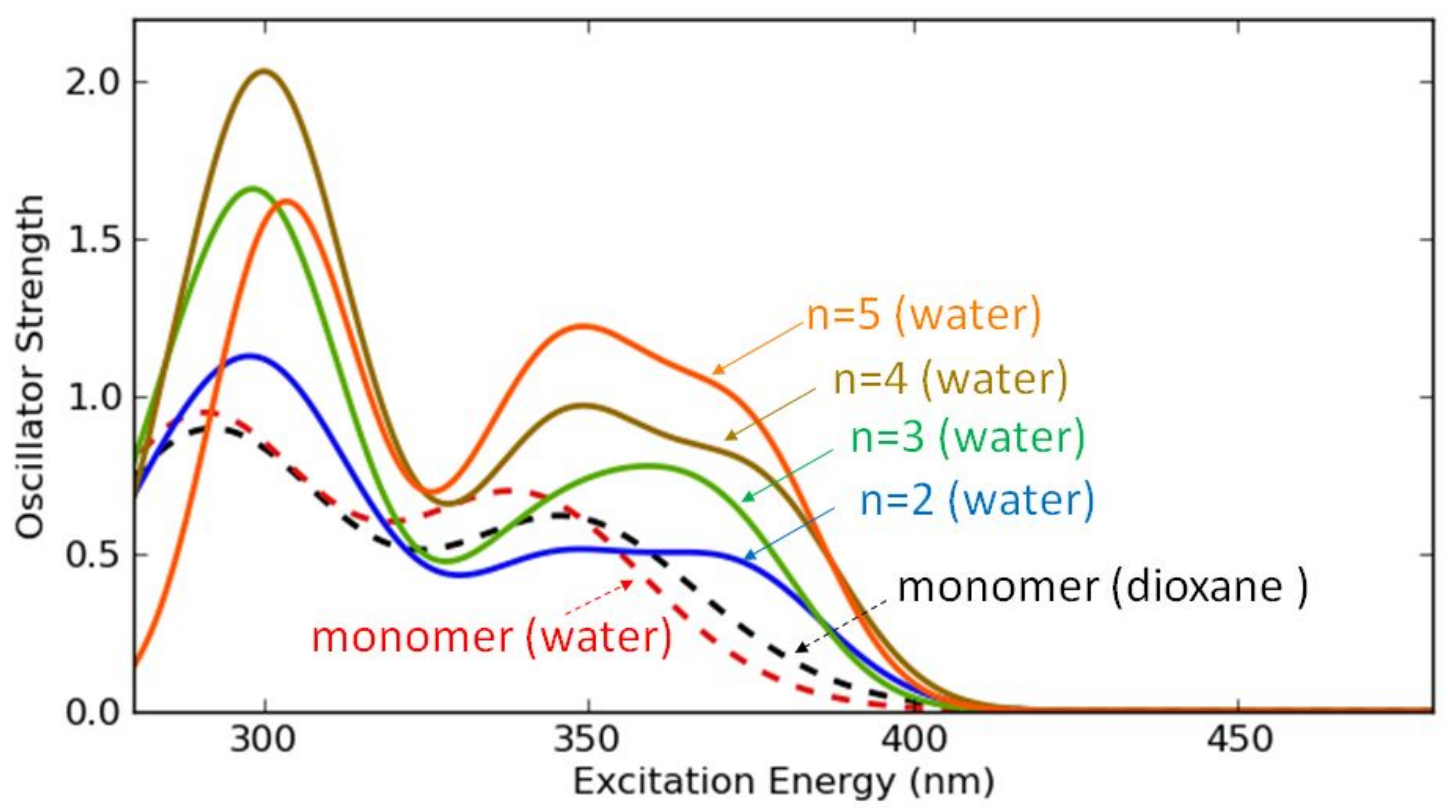

Figure S3. Calculated UV/Vis spectrum of the stable MBBT complexes in water in dependence on number of units in comparison with those of MBBT monomer in dioxane (black dashed) and water (red dashed). (TD PBE0-D3/6-31G(d,p)/PBF).

Optimized structure of the water stable MBBT-dimer 1. (PBE0-D3/6-31G(d,p)/PBF $\left(\mathrm{H}_{2} \mathrm{O}\right)$.

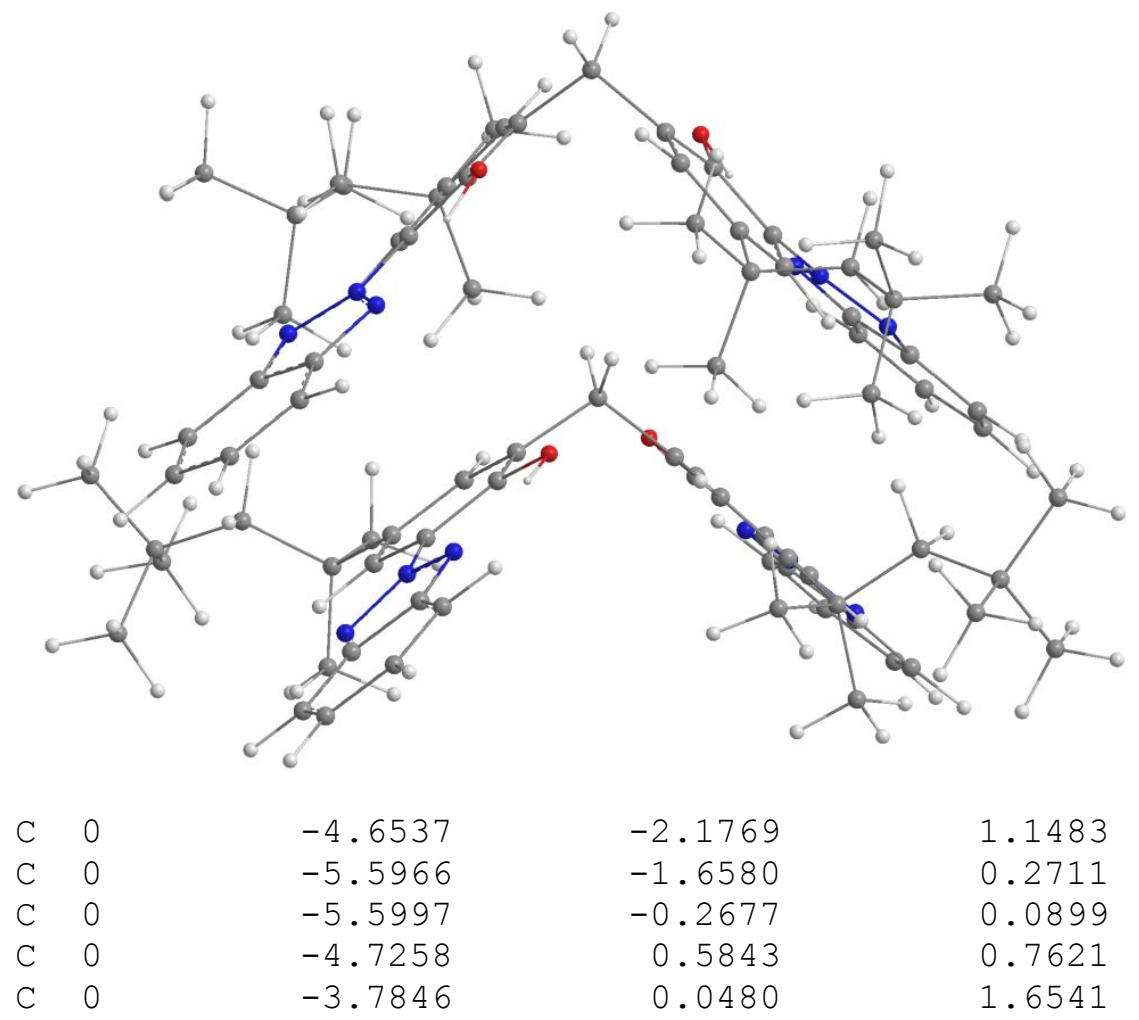




\begin{tabular}{|c|c|c|c|c|}
\hline C & 0 & -3.7554 & -1.3522 & 1.8140 \\
\hline $\mathrm{C}$ & 0 & -3.7710 & 2.3473 & -0.6942 \\
\hline $\mathrm{C}$ & 0 & -2.3917 & 2.4320 & -0.5194 \\
\hline $\mathrm{C}$ & 0 & -1.4935 & 2.5754 & -1.5862 \\
\hline $\mathrm{C}$ & 0 & -2.0412 & 2.6709 & -2.8574 \\
\hline $\mathrm{C}$ & 0 & -3.4161 & 2.5930 & -3.0621 \\
\hline $\mathrm{C}$ & 0 & -4.3145 & 2.4200 & -1.9887 \\
\hline $\mathrm{C}$ & 0 & -4.6869 & 2.0720 & 0.4812 \\
\hline 0 & 0 & -5.6460 & 2.3027 & -2.1317 \\
\hline 0 & 0 & -2.9449 & 0.8868 & 2.2812 \\
\hline $\mathrm{N}$ & 0 & -3.8837 & 2.6602 & -4.3986 \\
\hline $\mathrm{N}$ & 0 & -2.7676 & -1.9637 & 2.6262 \\
\hline $\mathrm{N}$ & 0 & -2.6374 & -3.2716 & 2.7152 \\
\hline $\mathrm{C}$ & 0 & -1.5701 & -3.4253 & 3.5261 \\
\hline $\mathrm{C}$ & 0 & -1.0925 & -2.1394 & 3.8913 \\
\hline $\mathrm{N}$ & 0 & -1.8872 & -1.2315 & 3.2958 \\
\hline $\mathrm{N}$ & 0 & -5.1672 & 2.4942 & -4.6873 \\
\hline $\mathrm{C}$ & 0 & -5.2162 & 2.6081 & -6.0284 \\
\hline $\mathrm{C}$ & 0 & -3.8931 & 2.8433 & -6.4846 \\
\hline $\mathrm{N}$ & 0 & -3.0710 & 2.8694 & -5.4152 \\
\hline $\mathrm{C}$ & 0 & -0.9400 & -4.5960 & 3.9924 \\
\hline $\mathrm{C}$ & 0 & 0.1528 & -4.4235 & 4.8113 \\
\hline $\mathrm{C}$ & 0 & 0.6292 & -3.1326 & 5.1756 \\
\hline $\mathrm{C}$ & 0 & 0.0263 & -1.9774 & 4.7309 \\
\hline C & 0 & -6.2980 & 2.5196 & -6.9257 \\
\hline $\mathrm{C}$ & 0 & -6.0007 & 2.6706 & -8.2619 \\
\hline $\mathrm{C}$ & 0 & -4.6742 & 2.9060 & -8.7229 \\
\hline $\mathrm{C}$ & 0 & -3.6080 & 2.9970 & -7.8566 \\
\hline $\mathrm{C}$ & 0 & -6.5531 & -2.6258 & -0.4448 \\
\hline $\mathrm{C}$ & 0 & -7.3502 & -1.8799 & -1.5200 \\
\hline $\mathrm{C}$ & 0 & -5.7133 & -3.7063 & -1.1483 \\
\hline $\mathrm{C}$ & 0 & -7.4928 & -3.1878 & 0.6649 \\
\hline $\mathrm{C}$ & 0 & -8.3849 & -4.4460 & 0.4989 \\
\hline $\mathrm{C}$ & 0 & -9.3468 & -4.4353 & 1.6984 \\
\hline $\mathrm{C}$ & 0 & -9.2233 & -4.4585 & -0.7812 \\
\hline $\mathrm{C}$ & 0 & -7.5636 & -5.7410 & 0.5817 \\
\hline $\mathrm{C}$ & 0 & 0.0316 & 2.6311 & -1.3981 \\
\hline $\mathrm{C}$ & 0 & 0.4218 & 1.8993 & -0.1048 \\
\hline $\mathrm{C}$ & 0 & 0.7170 & 1.8969 & -2.5621 \\
\hline $\mathrm{C}$ & 0 & 0.3826 & 4.1462 & -1.3452 \\
\hline $\mathrm{C}$ & 0 & 1.8211 & 4.7159 & -1.4504 \\
\hline $\mathrm{C}$ & 0 & 1.7013 & 6.2028 & -1.0786 \\
\hline $\mathrm{C}$ & 0 & 2.8226 & 4.0651 & -0.4927 \\
\hline $\mathrm{C}$ & 0 & 2.3690 & 4.6471 & -2.8828 \\
\hline $\mathrm{H}$ & 0 & -4.5841 & -3.2421 & 1.3343 \\
\hline $\mathrm{H}$ & 0 & -6.2811 & 0.1937 & -0.6158 \\
\hline $\mathrm{H}$ & 0 & -2.0249 & 2.3423 & 0.4965 \\
\hline $\mathrm{H}$ & 0 & -1.4165 & 2.7997 & -3.7318 \\
\hline $\mathrm{H}$ & 0 & -4.3225 & 2.6058 & 1.3631 \\
\hline $\mathrm{H}$ & 0 & -5.6940 & 2.4334 & 0.2670 \\
\hline $\mathrm{H}$ & 0 & -5.8638 & 2.3492 & -3.0917 \\
\hline $\mathrm{H}$ & 0 & -2.3440 & 0.3439 & 2.8474 \\
\hline $\mathrm{H}$ & 0 & -1.3030 & -5.5808 & 3.7132 \\
\hline $\mathrm{H}$ & 0 & 0.6758 & -5.2960 & 5.1943 \\
\hline $\mathrm{H}$ & 0 & 1.4990 & -3.0642 & 5.8240 \\
\hline $\mathrm{H}$ & 0 & 0.3896 & -0.9912 & 5.0056 \\
\hline $\mathrm{H}$ & 0 & -7.3098 & 2.3374 & -6.5755 \\
\hline $\mathrm{H}$ & 0 & -6.8008 & 2.6070 & -8.9949 \\
\hline $\mathrm{H}$ & 0 & -4.5063 & 3.0137 & -9.7912 \\
\hline
\end{tabular}




\begin{tabular}{|c|c|c|c|c|}
\hline $\mathrm{H}$ & 0 & -2.5940 & 3.1741 & -8.2039 \\
\hline $\mathrm{H}$ & 0 & -7.9677 & -2.5663 & -2.1013 \\
\hline $\mathrm{H}$ & 0 & -6.6810 & -1.3634 & -2.2171 \\
\hline $\mathrm{H}$ & 0 & -8.0141 & -1.1388 & -1.0673 \\
\hline $\mathrm{H}$ & 0 & -6.3468 & -4.3643 & -1.7516 \\
\hline $\mathrm{H}$ & 0 & -5.1558 & -4.3326 & -0.4458 \\
\hline $\mathrm{H}$ & 0 & -4.9896 & -3.2331 & -1.8214 \\
\hline $\mathrm{H}$ & 0 & -8.1422 & -2.3495 & 0.9558 \\
\hline $\mathrm{H}$ & 0 & -6.8762 & -3.3810 & 1.5516 \\
\hline $\mathrm{H}$ & 0 & -9.9740 & -5.3350 & 1.7044 \\
\hline $\mathrm{H}$ & 0 & -10.0093 & -3.5621 & 1.6669 \\
\hline $\mathrm{H}$ & 0 & -8.7976 & -4.4050 & 2.6477 \\
\hline $\mathrm{H}$ & 0 & -9.9297 & -5.2975 & -0.7582 \\
\hline $\mathrm{H}$ & 0 & -8.6030 & -4.5803 & -1.6742 \\
\hline $\mathrm{H}$ & 0 & -9.8024 & -3.5349 & -0.8897 \\
\hline $\mathrm{H}$ & 0 & -8.2318 & -6.6096 & 0.6263 \\
\hline $\mathrm{H}$ & 0 & -6.9410 & -5.7532 & 1.4849 \\
\hline $\mathrm{H}$ & 0 & -6.9066 & -5.8768 & -0.2800 \\
\hline $\mathrm{H}$ & 0 & 1.5025 & 1.7553 & -0.0448 \\
\hline $\mathrm{H}$ & 0 & -0.0493 & 0.9107 & -0.0650 \\
\hline $\mathrm{H}$ & 0 & 0.1197 & 2.4501 & 0.7894 \\
\hline $\mathrm{H}$ & 0 & 1.7892 & 1.7918 & -2.3700 \\
\hline $\mathrm{H}$ & 0 & 0.6062 & 2.4150 & -3.5192 \\
\hline $\mathrm{H}$ & 0 & 0.2980 & 0.8901 & -2.6716 \\
\hline $\mathrm{H}$ & 0 & -0.0581 & 4.5236 & -0.4102 \\
\hline $\mathrm{H}$ & 0 & -0.1950 & 4.6333 & -2.1420 \\
\hline $\mathrm{H}$ & 0 & 2.6682 & 6.7112 & -1.1752 \\
\hline $\mathrm{H}$ & 0 & 1.3580 & 6.3237 & -0.0445 \\
\hline $\mathrm{H}$ & 0 & 0.9827 & 6.7161 & -1.7294 \\
\hline $\mathrm{H}$ & 0 & 3.7740 & 4.6112 & -0.5168 \\
\hline $\mathrm{H}$ & 0 & 3.0353 & 3.0264 & -0.7642 \\
\hline $\mathrm{H}$ & 0 & 2.4569 & 4.0830 & 0.5406 \\
\hline $\mathrm{H}$ & 0 & 3.3018 & 5.2193 & -2.9559 \\
\hline $\mathrm{H}$ & 0 & 1.6566 & 5.0783 & -3.5967 \\
\hline $\mathrm{H}$ & 0 & 2.5863 & 3.6253 & -3.2005 \\
\hline C & 0 & -7.9609 & 1.4434 & 2.5077 \\
\hline $\mathrm{C}$ & 0 & -8.6056 & 2.5564 & 1.9797 \\
\hline $\mathrm{C}$ & 0 & -8.2467 & 3.8036 & 2.5072 \\
\hline $\mathrm{C}$ & 0 & -7.2802 & 3.9623 & 3.4986 \\
\hline C & 0 & -6.6701 & 2.8281 & 4.0539 \\
\hline $\mathrm{C}$ & 0 & -7.0349 & 1.5706 & 3.5368 \\
\hline $\mathrm{C}$ & 0 & -5.4344 & 5.5323 & 3.1584 \\
\hline $\mathrm{C}$ & 0 & -4.2100 & 5.3572 & 3.8026 \\
\hline $\mathrm{C}$ & 0 & -2.9867 & 5.3249 & 3.1186 \\
\hline $\mathrm{C}$ & 0 & -3.0323 & 5.4887 & 1.7381 \\
\hline C & 0 & -4.2361 & 5.7119 & 1.0808 \\
\hline $\mathrm{C}$ & 0 & -5.4603 & 5.7446 & 1.7717 \\
\hline C & 0 & -6.7526 & 5.3319 & 3.8803 \\
\hline 0 & 0 & -6.6443 & 5.9615 & 1.1682 \\
\hline 0 & 0 & -5.7713 & 2.9947 & 5.0435 \\
\hline $\mathrm{N}$ & 0 & -4.2099 & 5.8561 & -0.3258 \\
\hline $\mathrm{N}$ & 0 & -6.4471 & 0.3917 & 4.0529 \\
\hline $\mathrm{N}$ & 0 & -6.9528 & -0.8030 & 3.8203 \\
\hline $\mathrm{C}$ & 0 & -6.0987 & -1.6301 & 4.4572 \\
\hline $\mathrm{C}$ & 0 & -5.0905 & -0.8459 & 5.0787 \\
\hline $\mathrm{N}$ & 0 & -5.3480 & 0.4442 & 4.7935 \\
\hline $\mathrm{N}$ & 0 & -5.3121 & 5.6963 & -1.0457 \\
\hline C & 0 & -4.8895 & 5.8405 & -2.3151 \\
\hline $\mathrm{C}$ & 0 & -3.4911 & 6.0885 & -2.2748 \\
\hline
\end{tabular}




\begin{tabular}{|c|c|c|c|c|}
\hline $\mathrm{N}$ & 0 & -3.0954 & 6.0996 & -0.9852 \\
\hline $\mathrm{C}$ & 0 & -6.1008 & -3.0341 & 4.5756 \\
\hline $\mathrm{C}$ & 0 & -5.0825 & -3.5956 & 5.3098 \\
\hline $\mathrm{C}$ & 0 & -4.0733 & -2.8065 & 5.9303 \\
\hline $\mathrm{C}$ & 0 & -4.0565 & -1.4341 & 5.8325 \\
\hline $\mathrm{C}$ & 0 & -5.5919 & 5.7710 & -3.5328 \\
\hline $\mathrm{C}$ & 0 & -4.8527 & 5.9601 & -4.6780 \\
\hline $\mathrm{C}$ & 0 & -3.4521 & 6.2108 & -4.6419 \\
\hline $\mathrm{C}$ & 0 & -2.7527 & 6.2811 & -3.4592 \\
\hline $\mathrm{C}$ & 0 & -9.5868 & 2.3814 & 0.8057 \\
\hline $\mathrm{C}$ & 0 & -10.1834 & 3.7347 & 0.4028 \\
\hline $\mathrm{C}$ & 0 & -8.7829 & 1.8432 & -0.3894 \\
\hline $\mathrm{C}$ & 0 & -10.7095 & 1.4104 & 1.2828 \\
\hline $\mathrm{C}$ & 0 & -11.6554 & 0.6301 & 0.3292 \\
\hline $\mathrm{C}$ & 0 & -12.7373 & 0.0189 & 1.2358 \\
\hline $\mathrm{C}$ & 0 & -12.3523 & 1.5006 & -0.7195 \\
\hline $\mathrm{C}$ & 0 & -10.9436 & -0.5355 & -0.3744 \\
\hline $\mathrm{C}$ & 0 & -1.6400 & 5.0029 & 3.7913 \\
\hline $\mathrm{C}$ & 0 & -1.8038 & 4.8434 & 5.3029 \\
\hline $\mathrm{C}$ & 0 & -1.1868 & 3.6487 & 3.2159 \\
\hline $\mathrm{C}$ & 0 & -0.6810 & 6.1698 & 3.4175 \\
\hline $\mathrm{C}$ & 0 & 0.7919 & 6.3027 & 3.8858 \\
\hline $\mathrm{C}$ & 0 & 1.3650 & 7.4858 & 3.0858 \\
\hline $\mathrm{C}$ & 0 & 0.9200 & 6.6568 & 5.3729 \\
\hline $\mathrm{C}$ & 0 & 1.6440 & 5.0667 & 3.5783 \\
\hline $\mathrm{H}$ & 0 & -8.1433 & 0.4453 & 2.1253 \\
\hline $\mathrm{H}$ & 0 & -8.6875 & 4.7076 & 2.1074 \\
\hline $\mathrm{H}$ & 0 & -4.2371 & 5.1893 & 4.8735 \\
\hline $\mathrm{H}$ & 0 & -2.1422 & 5.4186 & 1.1236 \\
\hline $\mathrm{H}$ & 0 & -6.5962 & 5.4063 & 4.9609 \\
\hline $\mathrm{H}$ & 0 & -7.4791 & 6.0989 & 3.5934 \\
\hline $\mathrm{H}$ & 0 & -6.5030 & 5.8862 & 0.1928 \\
\hline $\mathrm{H}$ & 0 & -5.3307 & 2.1227 & 5.1958 \\
\hline $\mathrm{H}$ & 0 & -6.8723 & -3.6372 & 4.1077 \\
\hline $\mathrm{H}$ & 0 & -5.0375 & -4.6756 & 5.4219 \\
\hline $\mathrm{H}$ & 0 & -3.2906 & -3.3105 & 6.4909 \\
\hline $\mathrm{H}$ & 0 & -3.2825 & -0.8309 & 6.2975 \\
\hline $\mathrm{H}$ & 0 & -6.6576 & 5.5657 & -3.5591 \\
\hline $\mathrm{H}$ & 0 & -5.3461 & 5.9069 & -5.6449 \\
\hline $\mathrm{H}$ & 0 & -2.9249 & 6.3431 & -5.5830 \\
\hline $\mathrm{H}$ & 0 & -1.6838 & 6.4701 & -3.4324 \\
\hline $\mathrm{H}$ & 0 & -10.8743 & 3.6178 & -0.4336 \\
\hline $\mathrm{H}$ & 0 & -9.3990 & 4.4297 & 0.0832 \\
\hline $\mathrm{H}$ & 0 & -10.7340 & 4.1956 & 1.2309 \\
\hline $\mathrm{H}$ & 0 & -9.3983 & 1.8017 & -1.2937 \\
\hline $\mathrm{H}$ & 0 & -8.4034 & 0.8369 & -0.1967 \\
\hline $\mathrm{H}$ & 0 & -7.9251 & 2.4894 & -0.6013 \\
\hline $\mathrm{H}$ & 0 & -11.3420 & 1.9966 & 1.9652 \\
\hline $\mathrm{H}$ & 0 & -10.2486 & 0.6411 & 1.9143 \\
\hline $\mathrm{H}$ & 0 & -13.4336 & -0.5937 & 0.6506 \\
\hline $\mathrm{H}$ & 0 & -13.3177 & 0.7984 & 1.7435 \\
\hline $\mathrm{H}$ & 0 & -12.2906 & -0.6238 & 2.0046 \\
\hline $\mathrm{H}$ & 0 & -13.0989 & 0.9059 & -1.2601 \\
\hline $\mathrm{H}$ & 0 & -11.6502 & 1.8938 & -1.4616 \\
\hline $\mathrm{H}$ & 0 & -12.8711 & 2.3465 & -0.2543 \\
\hline $\mathrm{H}$ & 0 & -11.6798 & -1.1918 & -0.8543 \\
\hline $\mathrm{H}$ & 0 & -10.3795 & -1.1448 & 0.3429 \\
\hline $\mathrm{H}$ & 0 & -10.2511 & -0.2022 & -1.1498 \\
\hline $\mathrm{H}$ & 0 & -0.8604 & 4.5259 & 5.7533 \\
\hline
\end{tabular}




$\begin{array}{lllll}\mathrm{H} & \mathrm{O} & -2.5449 & 4.0706 & 5.5362 \\ \mathrm{H} & \mathrm{O} & -2.1147 & 5.7780 & 5.7826 \\ \mathrm{H} & 0 & -0.3214 & 3.2452 & 3.7470 \\ \mathrm{H} & 0 & -0.9177 & 3.7373 & 2.1590 \\ \mathrm{H} & 0 & -2.0003 & 2.9203 & 3.2848 \\ \mathrm{H} & 0 & -1.1876 & 7.0996 & 3.7149 \\ \mathrm{H} & 0 & -0.6430 & 6.1992 & 2.3206 \\ \mathrm{H} & 0 & 2.4010 & 7.6893 & 3.3814 \\ \mathrm{H} & 0 & 0.7799 & 8.3983 & 3.2536 \\ \mathrm{H} & 0 & 1.3598 & 7.2784 & 2.0093 \\ \mathrm{H} & 0 & 1.9585 & 6.9228 & 5.6055 \\ \mathrm{H} & 0 & 0.6405 & 5.8283 & 6.0279 \\ \mathrm{H} & 0 & 0.2898 & 7.5178 & 5.6272 \\ \mathrm{H} & 0 & 2.7081 & 5.2999 & 3.7089 \\ \mathrm{H} & 0 & 1.4982 & 4.7308 & 2.5443 \\ \mathrm{H} & 0 & 1.4090 & 4.2288 & 4.2414\end{array}$

\section{Structure of the optimised in water stable MBBT-dimer 2 .} (PBE0-D3/6-31G (d, P) / PBF (H2O).

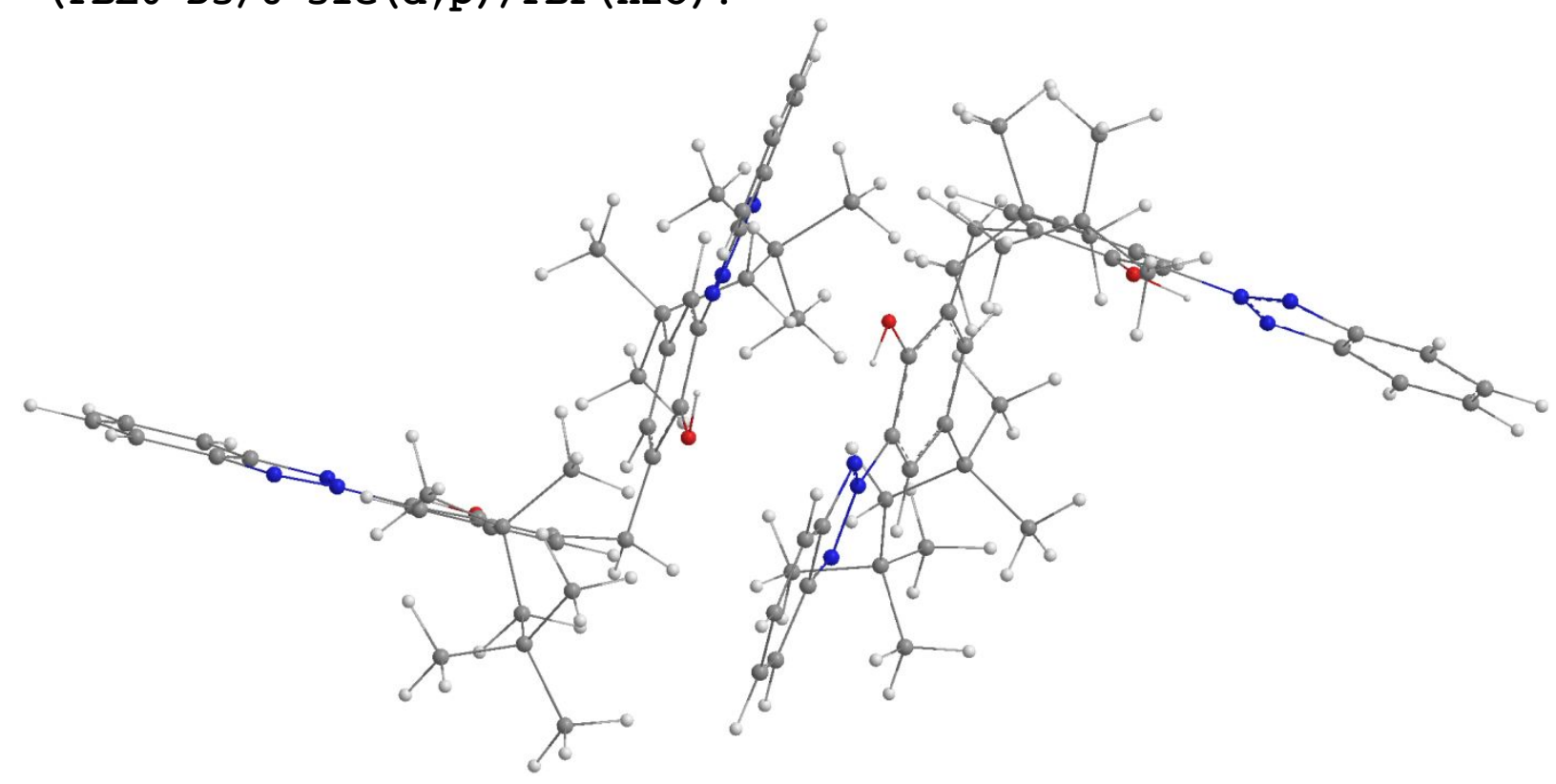

$\begin{array}{lllrr}\text { C } & 0 & 3.3062 & -2.6403 & 4.4004 \\ \mathrm{C} & 0 & 4.6608 & -2.9236 & 4.3244 \\ \mathrm{C} & 0 & 5.2248 & -3.0015 & 3.0396 \\ \mathrm{C} & 0 & 4.5064 & -2.7435 & 1.8794 \\ \mathrm{C} & 0 & 3.1299 & -2.4624 & 1.9641 \\ \mathrm{C} & 0 & 2.5470 & -2.4258 & 3.2461 \\ \mathrm{C} & 0 & 5.4223 & -1.1620 & 0.1935 \\ \mathrm{C} & 0 & 4.7362 & -0.5166 & -0.8342 \\ \mathrm{C} & 0 & 4.8694 & 0.8556 & -1.0921 \\ \mathrm{C} & 0 & 5.7127 & 1.5797 & -0.2583 \\ \mathrm{C} & 0 & 6.4316 & 0.9552 & 0.7511 \\ \mathrm{C} & 0 & 6.3170 & -0.4269 & 0.9863 \\ \mathrm{C} & 0 & 5.1964 & -2.6218 & 0.5351 \\ \mathrm{O} & 0 & 7.0197 & -1.0729 & 1.9305 \\ \mathrm{O} & 0 & 2.4627 & -2.2371 & 0.8181\end{array}$




\begin{tabular}{|c|c|c|c|c|}
\hline $\mathrm{N}$ & 0 & 7.2703 & 1.7523 & 1.5677 \\
\hline $\mathrm{N}$ & 0 & 1.1715 & -2.1203 & 3.4024 \\
\hline $\mathrm{N}$ & 0 & 0.5896 & -2.0779 & 4.5849 \\
\hline $\mathrm{C}$ & 0 & -0.6834 & -1.7330 & 4.2963 \\
\hline $\mathrm{C}$ & 0 & -0.8006 & -1.5824 & 2.8901 \\
\hline $\mathrm{N}$ & 0 & 0.4086 & -1.8390 & 2.3548 \\
\hline $\mathrm{N}$ & 0 & 7.7237 & 1.2888 & 2.7258 \\
\hline $\mathrm{C}$ & 0 & 8.4208 & 2.3206 & 3.2368 \\
\hline $\mathrm{C}$ & 0 & 8.3335 & 3.3924 & 2.3097 \\
\hline $\mathrm{N}$ & 0 & 7.5927 & 2.9888 & 1.2567 \\
\hline $\mathrm{C}$ & 0 & -1.7868 & -1.5251 & 5.1482 \\
\hline $\mathrm{C}$ & 0 & -2.9725 & -1.1700 & 4.5454 \\
\hline $\mathrm{C}$ & 0 & -3.0863 & -1.0200 & 3.1337 \\
\hline $\mathrm{C}$ & 0 & -2.0205 & -1.2205 & 2.2846 \\
\hline $\mathrm{C}$ & 0 & 9.1233 & 2.4504 & 4.4502 \\
\hline $\mathrm{C}$ & 0 & 9.7192 & 3.6687 & 4.6859 \\
\hline $\mathrm{C}$ & 0 & 9.6390 & 4.7424 & 3.7558 \\
\hline $\mathrm{C}$ & 0 & 8.9571 & 4.6298 & 2.5646 \\
\hline $\mathrm{C}$ & 0 & 5.5458 & -3.0849 & 5.5721 \\
\hline $\mathrm{C}$ & 0 & 6.7140 & -2.0876 & 5.4353 \\
\hline $\mathrm{C}$ & 0 & 4.7658 & -2.7233 & 6.8383 \\
\hline $\mathrm{C}$ & 0 & 6.0318 & -4.5637 & 5.5754 \\
\hline $\mathrm{C}$ & 0 & 6.7597 & -5.2366 & 6.7708 \\
\hline $\mathrm{C}$ & 0 & 7.3448 & -6.5480 & 6.2207 \\
\hline $\mathrm{C}$ & 0 & 7.9135 & -4.4081 & 7.3417 \\
\hline $\mathrm{C}$ & 0 & 5.7941 & -5.6127 & 7.9039 \\
\hline $\mathrm{C}$ & 0 & 4.1280 & 1.6027 & -2.2126 \\
\hline $\mathrm{C}$ & 0 & 3.2952 & 0.6375 & -3.0552 \\
\hline $\mathrm{C}$ & 0 & 3.1719 & 2.6058 & -1.5377 \\
\hline $\mathrm{C}$ & 0 & 5.2387 & 2.3174 & -3.0442 \\
\hline $\mathrm{C}$ & 0 & 5.0143 & 2.9978 & -4.4197 \\
\hline $\mathrm{C}$ & 0 & 6.2746 & 3.8425 & -4.6731 \\
\hline $\mathrm{C}$ & 0 & 4.9003 & 1.9811 & -5.5638 \\
\hline $\mathrm{C}$ & 0 & 3.8058 & 3.9360 & -4.4540 \\
\hline $\mathrm{H}$ & 0 & 2.7964 & -2.5586 & 5.3518 \\
\hline $\mathrm{H}$ & 0 & 6.2826 & -3.2108 & 2.9291 \\
\hline $\mathrm{H}$ & 0 & 4.0587 & -1.1181 & -1.4318 \\
\hline $\mathrm{H}$ & 0 & 5.8382 & 2.6499 & -0.3699 \\
\hline $\mathrm{H}$ & 0 & 4.5983 & -3.0892 & -0.2534 \\
\hline $\mathrm{H}$ & 0 & 6.1587 & -3.1401 & 0.5790 \\
\hline $\mathrm{H}$ & 0 & 7.4378 & -0.3945 & 2.5104 \\
\hline $\mathrm{H}$ & 0 & 1.5289 & -2.0021 & 1.0379 \\
\hline $\mathrm{H}$ & 0 & -1.6963 & -1.6391 & 6.2247 \\
\hline $\mathrm{H}$ & 0 & -3.8523 & -0.9955 & 5.1589 \\
\hline $\mathrm{H}$ & 0 & -4.0503 & -0.7368 & 2.7191 \\
\hline $\mathrm{H}$ & 0 & -2.1061 & -1.1049 & 1.2081 \\
\hline $\mathrm{H}$ & 0 & 9.1888 & 1.6296 & 5.1583 \\
\hline $\mathrm{H}$ & 0 & 10.2760 & 3.8204 & 5.6068 \\
\hline $\mathrm{H}$ & 0 & 10.1349 & 5.6780 & 4.0005 \\
\hline $\mathrm{H}$ & 0 & 8.8945 & 5.4494 & 1.8545 \\
\hline $\mathrm{H}$ & 0 & 7.2996 & -2.0248 & 6.3555 \\
\hline $\mathrm{H}$ & 0 & 6.3275 & -1.0868 & 5.2145 \\
\hline $\mathrm{H}$ & 0 & 7.3955 & -2.3666 & 4.6269 \\
\hline $\mathrm{H}$ & 0 & 5.4282 & -2.7317 & 7.7076 \\
\hline $\mathrm{H}$ & 0 & 3.9451 & -3.4210 & 7.0352 \\
\hline $\mathrm{H}$ & 0 & 4.3483 & -1.7132 & 6.7596 \\
\hline $\mathrm{H}$ & 0 & 6.6871 & -4.6709 & 4.7008 \\
\hline $\mathrm{H}$ & 0 & 5.1537 & -5.1887 & 5.3625 \\
\hline $\mathrm{H}$ & 0 & 7.8168 & -7.1286 & 7.0222 \\
\hline
\end{tabular}




\begin{tabular}{|c|c|c|c|c|}
\hline $\mathrm{H}$ & 0 & 8.1050 & -6.3513 & 5.4562 \\
\hline $\mathrm{H}$ & 0 & 6.5645 & -7.1718 & 5.7675 \\
\hline $\mathrm{H}$ & 0 & 8.4890 & -5.0103 & 8.0553 \\
\hline $\mathrm{H}$ & 0 & 7.5573 & -3.5229 & 7.8768 \\
\hline $\mathrm{H}$ & 0 & 8.5965 & -4.0765 & 6.5525 \\
\hline $\mathrm{H}$ & 0 & 6.3169 & -6.2147 & 8.6572 \\
\hline $\mathrm{H}$ & 0 & 4.9573 & -6.2104 & 7.5224 \\
\hline $\mathrm{H}$ & 0 & 5.3795 & -4.7400 & 8.4128 \\
\hline $\mathrm{H}$ & 0 & 2.7068 & 1.1881 & -3.7939 \\
\hline $\mathrm{H}$ & 0 & 2.5886 & 0.0782 & -2.4318 \\
\hline $\mathrm{H}$ & 0 & 3.9261 & -0.0773 & -3.5914 \\
\hline $\mathrm{H}$ & 0 & 2.5424 & 3.1188 & -2.2691 \\
\hline $\mathrm{H}$ & 0 & 3.7188 & 3.3688 & -0.9731 \\
\hline $\mathrm{H}$ & 0 & 2.5128 & 2.0797 & -0.8375 \\
\hline $\mathrm{H}$ & 0 & 6.0489 & 1.5896 & -3.1861 \\
\hline $\mathrm{H}$ & 0 & 5.6632 & 3.0898 & -2.3904 \\
\hline $\mathrm{H}$ & 0 & 6.2506 & 4.2906 & -5.6738 \\
\hline $\mathrm{H}$ & 0 & 7.1832 & 3.2332 & -4.5972 \\
\hline $\mathrm{H}$ & 0 & 6.3590 & 4.6542 & -3.9404 \\
\hline $\mathrm{H}$ & 0 & 4.8719 & 2.5000 & -6.5295 \\
\hline $\mathrm{H}$ & 0 & 3.9998 & 1.3667 & -5.4936 \\
\hline $\mathrm{H}$ & 0 & 5.7650 & 1.3081 & -5.5773 \\
\hline $\mathrm{H}$ & 0 & 3.7984 & 4.5033 & -5.3928 \\
\hline $\mathrm{H}$ & 0 & 3.8364 & 4.6543 & -3.6271 \\
\hline $\mathrm{H}$ & 0 & 2.8610 & 3.3875 & -4.3955 \\
\hline $\mathrm{C}$ & 0 & 9.1570 & -1.0591 & -2.0556 \\
\hline $\mathrm{C}$ & 0 & 9.2026 & -0.1524 & -3.1068 \\
\hline $\mathrm{C}$ & 0 & 9.6401 & 1.1461 & -2.8085 \\
\hline $\mathrm{C}$ & 0 & 9.9786 & 1.5541 & -1.5192 \\
\hline $\mathrm{C}$ & 0 & 9.8453 & 0.6473 & -0.4560 \\
\hline $\mathrm{C}$ & 0 & 9.4635 & -0.6729 & -0.7589 \\
\hline $\mathrm{C}$ & 0 & 11.9592 & 2.8357 & -0.7077 \\
\hline $\mathrm{C}$ & 0 & 12.2265 & 2.9592 & 0.6487 \\
\hline $\mathrm{C}$ & 0 & 13.4809 & 2.6844 & 1.2217 \\
\hline $\mathrm{C}$ & 0 & 14.4923 & 2.3344 & 0.3431 \\
\hline C & 0 & 14.2689 & 2.2557 & -1.0352 \\
\hline $\mathrm{C}$ & 0 & 12.9993 & 2.4877 & -1.5917 \\
\hline $\mathrm{C}$ & 0 & 10.5398 & 2.9318 & -1.2311 \\
\hline 0 & 0 & 12.7170 & 2.3890 & -2.9038 \\
\hline 0 & 0 & 10.0911 & 1.0737 & 0.7925 \\
\hline $\mathrm{N}$ & 0 & 15.3667 & 1.8721 & -1.8442 \\
\hline $\mathrm{N}$ & 0 & 9.4149 & -1.6578 & 0.2574 \\
\hline $\mathrm{N}$ & 0 & 8.8904 & -2.8473 & 0.0593 \\
\hline $\mathrm{C}$ & 0 & 9.1115 & -3.4844 & 1.2273 \\
\hline $\mathrm{C}$ & 0 & 9.7841 & -2.5950 & 2.1060 \\
\hline $\mathrm{N}$ & 0 & 9.9630 & -1.4355 & 1.4453 \\
\hline $\mathrm{N}$ & 0 & 15.2150 & 1.5925 & -3.1310 \\
\hline $\mathrm{C}$ & 0 & 16.4505 & 1.2256 & -3.5295 \\
\hline $\mathrm{C}$ & 0 & 17.3071 & 1.3126 & -2.4010 \\
\hline $\mathrm{N}$ & 0 & 16.5799 & 1.7325 & -1.3459 \\
\hline C & 0 & 8.7999 & -4.8017 & 1.6161 \\
\hline $\mathrm{C}$ & 0 & 9.1954 & -5.1804 & 2.8790 \\
\hline $\mathrm{C}$ & 0 & 9.8739 & -4.2893 & 3.7568 \\
\hline $\mathrm{C}$ & 0 & 10.1725 & -2.9929 & 3.3996 \\
\hline $\mathrm{C}$ & 0 & 16.9306 & 0.8011 & -4.7837 \\
\hline C & 0 & 18.2673 & 0.4762 & -4.8493 \\
\hline $\mathrm{C}$ & 0 & 19.1273 & 0.5644 & -3.7179 \\
\hline $\mathrm{C}$ & 0 & 18.6740 & 0.9794 & -2.4861 \\
\hline $\mathrm{C}$ & 0 & 8.8624 & -0.6500 & -4.5209 \\
\hline
\end{tabular}




\begin{tabular}{|c|c|c|c|c|}
\hline C & 0 & 8.9138 & 0.5062 & -5.5246 \\
\hline C & 0 & 9.9504 & -1.6650 & -4.9156 \\
\hline $\mathrm{C}$ & 0 & 7.4251 & -1.2532 & -4.4541 \\
\hline C & 0 & 6.8420 & -2.2366 & -5.5015 \\
\hline $\mathrm{C}$ & 0 & 5.3586 & -2.3981 & -5.1301 \\
\hline $\mathrm{C}$ & 0 & 6.9264 & -1.7328 & -6.9439 \\
\hline $\mathrm{C}$ & 0 & 7.4778 & -3.6316 & -5.4100 \\
\hline $\mathrm{C}$ & 0 & 13.6382 & 2.6699 & 2.7579 \\
\hline $\mathrm{C}$ & 0 & 13.3070 & 4.0668 & 3.3000 \\
\hline $\mathrm{C}$ & 0 & 12.6077 & 1.6522 & 3.2877 \\
\hline $\mathrm{C}$ & 0 & 15.0798 & 2.2362 & 3.1202 \\
\hline $\mathrm{C}$ & 0 & 15.6865 & 2.2885 & 4.5509 \\
\hline C & 0 & 16.9639 & 1.4339 & 4.4807 \\
\hline $\mathrm{C}$ & 0 & 16.1096 & 3.7118 & 4.9421 \\
\hline $\mathrm{C}$ & 0 & 14.7989 & 1.7092 & 5.6553 \\
\hline $\mathrm{H}$ & 0 & 8.8878 & -2.0967 & -2.2160 \\
\hline $\mathrm{H}$ & 0 & 9.7542 & 1.8764 & -3.6033 \\
\hline $\mathrm{H}$ & 0 & 11.3898 & 3.2168 & 1.2896 \\
\hline $\mathrm{H}$ & 0 & 15.4847 & 2.0836 & 0.6877 \\
\hline $\mathrm{H}$ & 0 & 9.9186 & 3.4315 & -0.4806 \\
\hline $\mathrm{H}$ & 0 & 10.5006 & 3.5300 & -2.1464 \\
\hline $\mathrm{H}$ & 0 & 13.5142 & 2.0499 & -3.3739 \\
\hline $\mathrm{H}$ & 0 & 10.1489 & 0.2805 & 1.3743 \\
\hline $\mathrm{H}$ & 0 & 8.2845 & -5.4813 & 0.9436 \\
\hline $\mathrm{H}$ & 0 & 8.9917 & -6.1923 & 3.2189 \\
\hline $\mathrm{H}$ & 0 & 10.1691 & -4.6515 & 4.7381 \\
\hline $\mathrm{H}$ & 0 & 10.6922 & -2.3159 & 4.0713 \\
\hline $\mathrm{H}$ & 0 & 16.2750 & 0.7296 & -5.6467 \\
\hline $\mathrm{H}$ & 0 & 18.6869 & 0.1376 & -5.7930 \\
\hline $\mathrm{H}$ & 0 & 20.1721 & 0.2902 & -3.8384 \\
\hline $\mathrm{H}$ & 0 & 19.3256 & 1.0443 & -1.6194 \\
\hline $\mathrm{H}$ & 0 & 8.6673 & 0.1598 & -6.5296 \\
\hline $\mathrm{H}$ & 0 & 9.9149 & 0.9495 & -5.5678 \\
\hline $\mathrm{H}$ & 0 & 8.2028 & 1.2960 & -5.2603 \\
\hline $\mathrm{H}$ & 0 & 9.8359 & -1.9879 & -5.9557 \\
\hline $\mathrm{H}$ & 0 & 9.9370 & -2.5542 & -4.2782 \\
\hline $\mathrm{H}$ & 0 & 10.9374 & -1.1984 & -4.8180 \\
\hline $\mathrm{H}$ & 0 & 6.7427 & -0.3943 & -4.3897 \\
\hline $\mathrm{H}$ & 0 & 7.3105 & -1.7633 & -3.4915 \\
\hline $\mathrm{H}$ & 0 & 4.8627 & -3.1054 & -5.8053 \\
\hline $\mathrm{H}$ & 0 & 4.8171 & -1.4469 & -5.1815 \\
\hline $\mathrm{H}$ & 0 & 5.2526 & -2.7808 & -4.1074 \\
\hline $\mathrm{H}$ & 0 & 6.3751 & -2.4075 & -7.6103 \\
\hline $\mathrm{H}$ & 0 & 7.9599 & -1.6926 & -7.3032 \\
\hline $\mathrm{H}$ & 0 & 6.4895 & -0.7326 & -7.0436 \\
\hline $\mathrm{H}$ & 0 & 6.8999 & -4.3455 & -6.0091 \\
\hline $\mathrm{H}$ & 0 & 7.4817 & -3.9945 & -4.3747 \\
\hline $\mathrm{H}$ & 0 & 8.5051 & -3.6521 & -5.7783 \\
\hline $\mathrm{H}$ & 0 & 13.2679 & 4.0632 & 4.3937 \\
\hline $\mathrm{H}$ & 0 & 12.3284 & 4.4023 & 2.9467 \\
\hline $\mathrm{H}$ & 0 & 14.0568 & 4.7984 & 2.9820 \\
\hline $\mathrm{H}$ & 0 & 12.5939 & 1.6214 & 4.3782 \\
\hline $\mathrm{H}$ & 0 & 12.8355 & 0.6460 & 2.9177 \\
\hline $\mathrm{H}$ & 0 & 11.5962 & 1.9072 & 2.9619 \\
\hline $\mathrm{H}$ & 0 & 15.7695 & 2.8337 & 2.5095 \\
\hline $\mathrm{H}$ & 0 & 15.1870 & 1.2015 & 2.7654 \\
\hline $\mathrm{H}$ & 0 & 17.5144 & 1.4774 & 5.4280 \\
\hline $\mathrm{H}$ & 0 & 17.6327 & 1.7865 & 3.6861 \\
\hline $\mathrm{H}$ & 0 & 16.7249 & 0.3833 & 4.2764 \\
\hline
\end{tabular}




$\begin{array}{lllll}\mathrm{H} & 0 & 16.6714 & 3.6901 & 5.8839 \\ \mathrm{H} & 0 & 15.2568 & 4.3797 & 5.0834 \\ \mathrm{H} & 0 & 16.7606 & 4.1515 & 4.1767 \\ \mathrm{H} & 0 & 15.3685 & 1.6456 & 6.5908 \\ \mathrm{H} & 0 & 14.4519 & 0.7002 & 5.4063 \\ \mathrm{H} & 0 & 13.9240 & 2.3368 & 5.8505\end{array}$

